\title{
Light Makes an Impact on the Lives and Healthcare of Scots Carol Trager-Cowan
}

Early detection of breast cancer; noninvasive measurement of blood glucose; wide-angle, high-resolution imaging of the retina; early detection of tooth decay; novel "sticking plasters" for the treatment of skin cancer; and production of positron beams for positron emission tomography are but a few of the medicinal applications of lasers and light-emitting diodes recently developed or under development in Scotland. Scientists and engineers in Scotland's burgeoning "biophotonics" industry are exploiting the highest power lasers in the world and the newest organic light-emitting diodes to meet the needs of society.

The Oxford English Dictionary defines photonics as "the branch of technology that deals with the applications of the particle properties of light"; recently, "photonics" has become a general term used to describe the study and application of light. The term "biophotonics" is used to describe technologies where light is applied to biological material. The term "biophotonics" has yet to make it into the Oxford English Dictionary; however, this new subject is having a major impact on the lives of Scots.

Developments in biophotonics in Scotland are leading to new, more effective detection and treatment of cancer, diabetes, and eye disease than available before; they are helping to save sight and lives.

One of the researchers who has made a tremendous impact on the Scottish biophotonics industry is Douglas Anderson. Anderson developed a patient-friendly method of obtaining a wide-field image of the eye in a fast single measurement, after his five-year-old son Leif lost the sight in one eye because a retinal detachment was detected too late, despite regular eye examinations with conventional imaging instrumentation. Conventional imaging methods require the dilation of the eye with eye drops followed by the acquisition of multiple images-an uncomfortable procedure, particularly for a small child.

Anderson founded Optos in 1992 and in 1999 the Panoramic200 Scanning Laser Ophthalmoscope (P200) received FDA $510 \mathrm{k}$ clearance, so that it could be sold in the United States, and full CE marking, which allows sales in the European market. The P200 captures a detailed digital image of up to 200 degrees, or approximately $80 \%$ of the eye, in under a second. Conventional imaging techniques acquire images of only $\sim 5 \%$ of the eye. The ability to image such a large angular area allows

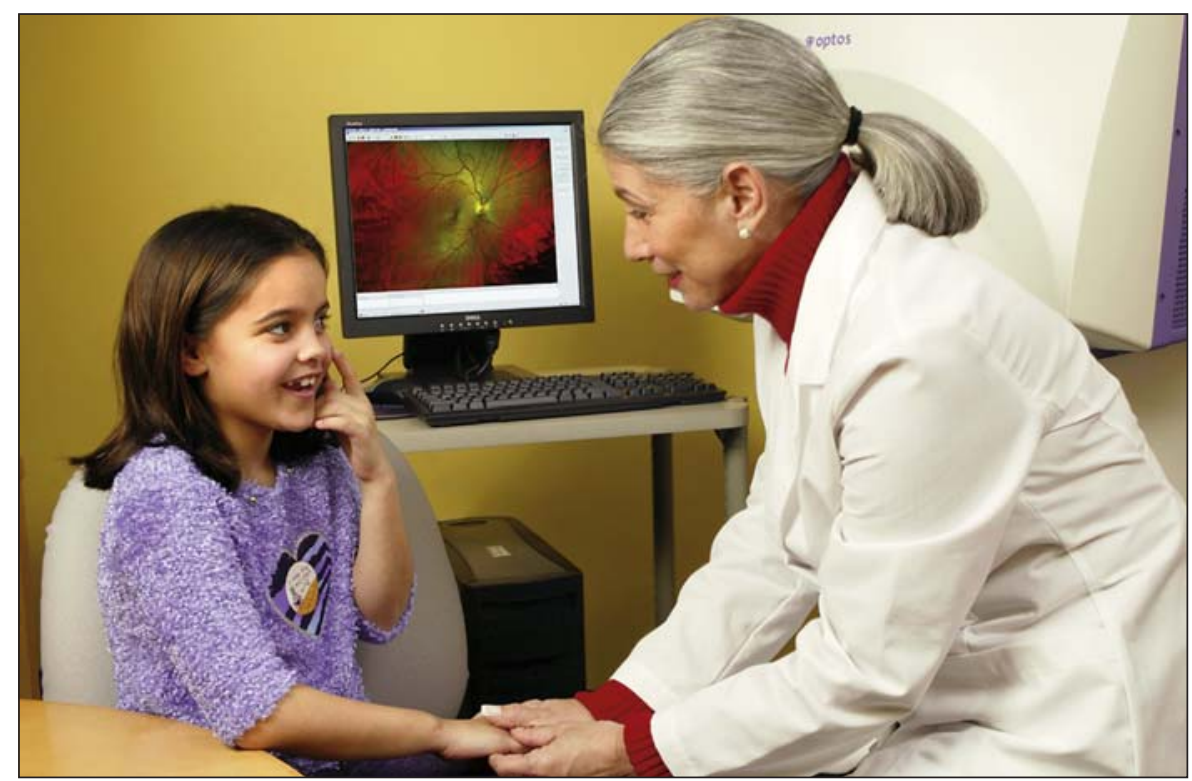

A physician and her patient use the Optos instrument which captures a digital image of $\sim 80 \%$ of the eye, enabling early detection of disease.

the early diagnosis of eye diseases such as glaucoma, diabetic retinopathy, and age-related macular degeneration and diseases such as diabetes, hypertension, and certain cancers.

Optos was floated on the London Stock Exchange on February 15, 2006. It has installed over 2500 instruments worldwide with revenues of around $\$ 70$ million.

In 2007, Optos received the Queen's Award for Innovation and the Scotland Plc Award for Innovation.

While Optos is now a well-established company and a beacon of the Scottish biophotonics industry, Lumicure is just at the embryonic stage of taking its innovative product to the market place. Lumicure was set up in 2004 by Ifor Samuel of St.

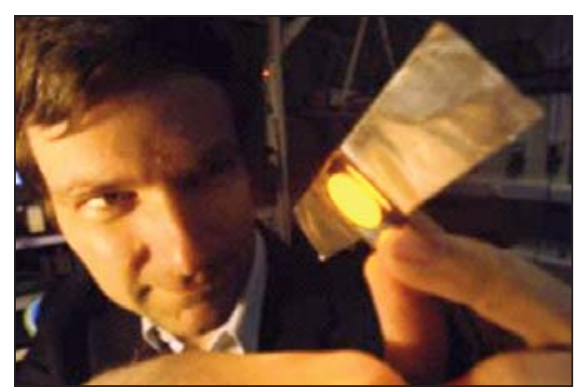

Ifor Samuel and his "optical sticking plaster" provides low-intensity light for photodynamic therapy of skin cancer. (Photo: Alan Richardson, Pix-AR)
Andrews University and James Ferguson, head of the photobiology unit at Ninewells Hospital in Dundee. The "optical sticking plaster" is expected to revolutionize the use of photodynamic therapy in the treatment of skin cancer. Skin cancer will affect $20 \%$ of Americans and $50 \%$ of Australians in their lifetimes. Photodynamic therapy is a treatment of skin cancer which combines a photosensitizing (i.e., makes sensitive to light) drug with a specific wavelength of light to produce singlet oxygen within the cancerous tissue. The oxygen reacts with and destroys the cancer cells. Light is produced from a laser or from light-emitting diodes. The "optical sticking plaster" is a lightweight wearable device, based on a large area $(2 \mathrm{~cm}$ diameter-about the size of a skin cancer lesion) polymer organic light-emitting diode emitting in the orange-red region of the visible spectrum. It is powered by a small battery which can be slipped into a pocket. This device allows the patient to move around instead of having to remain still under a high-intensity light source for up to 30 minutes. The use of high-intensity light sources for conventional photodynamic therapy can be painful; however, with the "optical sticking plaster," a lowintensity light source can be applied comfortably over a few hours. The patient can also be treated at home after the application of the photosensitizing drug instead of having to remain in the hospital. The 
"optical sticking plaster" is offered as a safe, effective, inexpensive new approach to the treatment of skin cancer and is expected to be available for general use in about two years. As a final note to this tale, Samuel did not set out to develop organic light-emitting diodes for medical applications-his research was, in fact, originally aimed at developing light sources for advanced displays. In 2007, Samuel was awarded the Beilby medal and prize for his distinguished contributions to the development of organic semiconductors for applications in displays, lasers, and medicine.

Light can be used to detect cancers as well as to treat them. Highland Innovation Centre Ltd, set up in 2000, has developed an optical instrument-the BreastChecker-which women can use at home to monitor themselves for breast cancer. The instrument utilizes a set of yellow light-emitting diodes to provide a bright light source which is shone through the underside of the breast. Blood vessels strongly absorb the yellow light and so are revealed as dark areas on the breast. The presence of a tumor stimulates the growth of many more blood vessels, leading to a chaotic mass of blood vessels surrounding the tumor, which will be revealed as dark areas by this instrument. The BreastChecker cannot be used to tell whether an observed dark area is indeed a cancerous tumor; however, it will alert the woman to see her doctor. The BreastChecker allows tumors to be detected while they are small and its availability is expected to reduce the need for mastectomies. The BreastChecker is currently under test and evaluation in Taiwan, Korea, Romania, Egypt, Cyprus, Portugal, the United States, and the United Kingdom. It is expected to be available to buy from local pharmacies for about $\$ 100$ within two years of trials being completed.

Another application which uses light as a non-invasive probe is the "optical nose" being developed by a research group at Glasgow University. Originally developed for oil prospecting, the optical nose uses a mid-infrared laser and absorption spectroscopy to measure trace levels of ethane gas in real time. The "optical nose" is sensitive enough to detect ethane in the breath at less than one part per billion. Cells in the body release ethane when they are damaged by free radicals. This process is occurring all of the time; however, during the onset of serious illnesses such as sepsis, or during renal dialysis, or during radiotherapy, cell damage is increased. Trials are presently underway monitoring intensive care patients to dis-

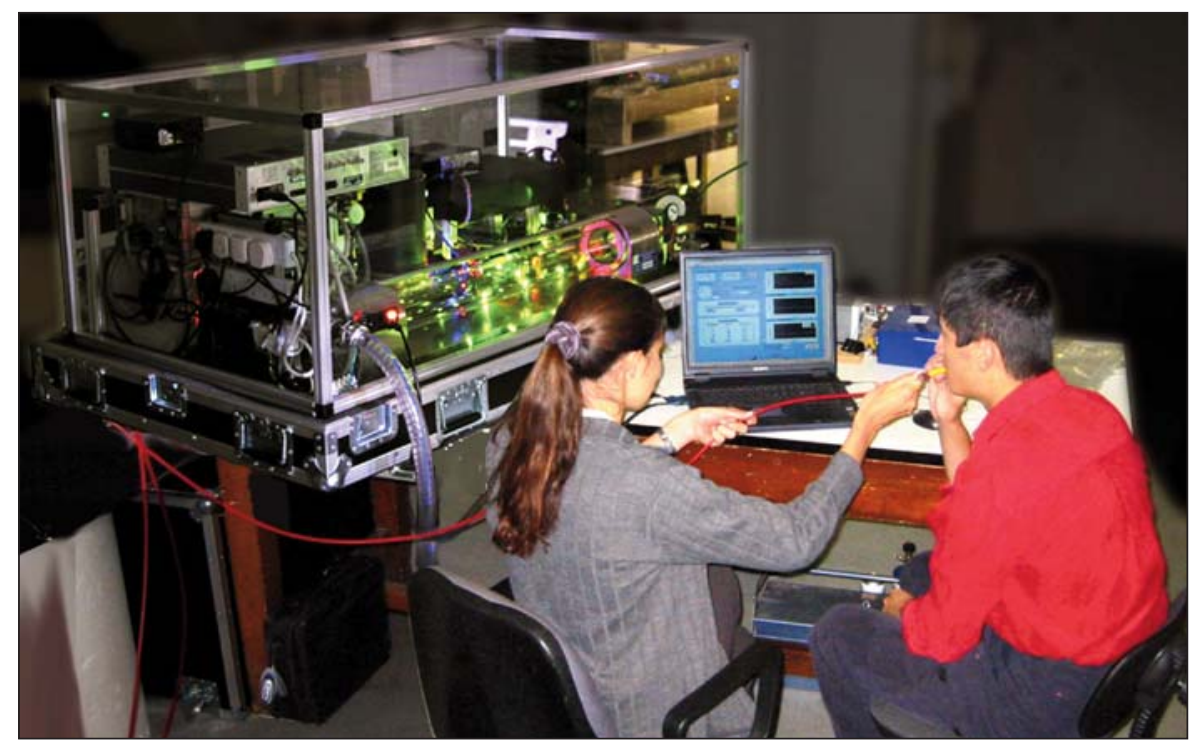

The "optical nose" undergoes testing for measuring trace levels of ethane gas in real time.

cover whether the "optical nose" can provide doctors with a new non-invasive healthcare technique. Leader of the project Kenneth Skeldon and colleagues at Glasgow University are also presently developing a more compact instrument by exploiting recently developed quantum cascade laser sources.

At the Institute of Photonics at Strathclyde University, John Girkin and colleagues are developing a scanning laser imaging system that can profile through tooth enamel to detect the early signs of tooth decay. A laser can be used to produce fluorescence from teeth, with healthy enamel fluorescing in the red, while diseased tissue fluoresces in the green. Using conventional techniques, early signs of tooth decay are hard to spot as the dentist is looking for a white spot on a white background. However, Girkin's laser technology is expected to detect tooth decay at a stage where the tooth can be healed through careful cleaning and application of fluoride-so the use of lasers may see the demise of the dentist's drill.

The Institute of Photonics is also working with Lein Applied Diagnostics who are developing an optical sensor that can be used by diabetics to painlessly determine their glucose levels. Red light is shone onto the eye and the light reflected from the aqueous humour-the liquid contained in the anterior chamber located between the cornea and the iris in the eye-is analyzed to determine glucose level.

In looking to the future, a team of researchers at Strathclyde University are developing a laser capable of generating

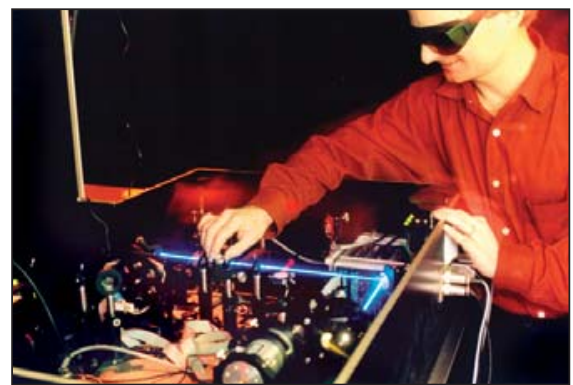

John Girkin and his laser system offers detection of the early stages of tooth decay.

intense, short pulses which when incident on a solid target can produce protons, electrons, neutrons, and positrons which can be used for medical applications. Positrons can be used in positron emission tomography to find tumors and protons can be used to treat tumors with greater efficiency than conventional radiotherapy. Proton beams allow specific targeting of tumors while minimizing damage to the surrounding healthy tissue.

In summary, biophotonics is an exciting new field expected to revolutionize how we detect, monitor, and treat disease.

\section{Acknowledgments}

I would like to thank Iain Ross, Chris Gracie, Kenneth Skeldon, and John Girkin for their help in writing this article.

Carol Trager-Cowan is a Senior Lecturer at the University of Strathclyde in Glasgow, Scotland. 